\title{
Comparative Genomics Approaches to Understanding Virulence and Antimicrobial Resistance of Salmonella Typhimurium ST1539 Isolated from a Poultry Slaughterhouse in Korea ${ }^{\mathrm{S}}$
}

\author{
Eunsuk Kim ${ }^{1}$, Soyeon Park ${ }^{2}$, Seongbeom Cho ${ }^{3}$, Tae-Wook Hahn ${ }^{2 *}$, and Hyunjin Yoon ${ }^{1,4 *}$ \\ ${ }^{1}$ Department of Molecular Science and Technology, Ajou University, Suwon 16499, Republic of Korea \\ ${ }^{2}$ College of Veterinary Medicine and Institute of Veterinary Science, Kangwon National University, Chuncheon 24341, Republic of Korea \\ ${ }^{3}$ Department of Veterinary Pathobiology and Preventive Medicine, Research Institute for Veterinary Science and College of Veterinary \\ Medicine, Seoul National University, Seoul 08826, Republic of Korea \\ ${ }^{4}$ Department of Applied Chemistry and Biological Engineering, Ajou University, Suwon 16499, Republic of Korea
}

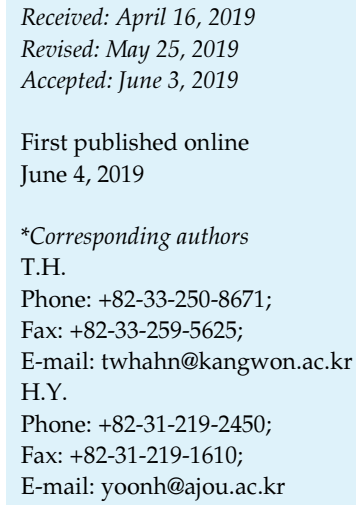

S upplementary data for this paper are available on-line only at http://jmb.or.kr.

pISSN 1017-7825, eISSN 1738-8872

Copyright(C) 2019 by

The Korean Society for Microbiology and Biotechnology

\begin{abstract}
Non-typhoidal Salmonella (NTS) is one of the most frequent causes of bacterial foodborne illnesses. Considering that the main reservoir of NTS is the intestinal tract of livestock, foods of animal origin are regarded as the main vehicles of Salmonella infection. In particular, poultry colonized with Salmonella Typhimurium (S. Typhimurium), a dominant serotype responsible for human infections, do not exhibit overt signs and symptoms, thereby posing a potential health risk to humans. In this study, comparative genomics approaches were applied to two S. Typhimurium strains, ST1539 and ST1120, isolated from a duck slaughterhouse and a pig farm, respectively, to characterize their virulence and antimicrobial resistance-associated genomic determinants. ST1539 containing a chromosome $(4,905,039 \mathrm{bp} ; 4,403$ CDSs) and a plasmid (93,876 bp; 96 CDSs) was phylogenetically distinct from other $S$. Typhimurium strains such as ST1120 and LT2. Compared to the ST1120 genome (previously deposited in GenBank; CP021909.1 and CP021910.1), ST1539 possesses more virulence determinants, including ST64B prophage, plasmid spv operon encoding virulence factors, genes encoding SseJ effector, Rck invasin, and biofilm-forming factors ( $b c f$ operon and pef $A B$ ). In accordance with the in silico prediction, ST1539 exhibited higher cytotoxicity against epithelial cells, better survival inside macrophage cells, and faster mice-killing activity than ST1120. However, ST1539 showed less resistance against antibiotics than ST1120, which may be attributed to the multiple resistanceassociated genes in the ST1120 chromosome. The accumulation of comparative genomics data on $S$. Typhimurium isolates from livestock would enrich our understanding of strategies Salmonella employs to adapt to diverse host animals.
\end{abstract}

Keywords: Salmonella Typhimurium, comparative genomics, virulence, antibiotic resistance

\section{Introduction}

Salmonellosis is a foodborne illness caused by nontyphoidal Salmonella (NTS) serotypes and typically induces a self-limiting gastroenteritis characterized by diarrhea, fever, and abdominal cramps within 4 and $72 \mathrm{~h}$ [1, 2]. However, an infection may be life-threatening due to bacteremia and extra-intestinal colonization in infants, the elderly, and immunocompromised patients [1, 2]. NTS colonizes animal and human intestines and is shed into soil and water through feces, circulating within agricultural ecosystems. In industrialized countries, human infections have been closely associated with contaminated foods of animal origin such as poultry, pork, and beef. Among more than 2,500 serotypes, $S$. Enteritidis and $S$. Typhimurium rank among the most notorious serovars responsible for human infections [3, 4]. Interestingly, S. Enteritidis is mainly isolated in poultry commodities, while $S$. Typhimurium is 
associated with a wide variety of animal commodities such as poultry, pork, and beef [4]. Due to the intimate correlation between contaminated poultry and human infection, poultry has been regarded as a primary vehicle transmitting NTS to humans. An additional risk factor in terms of poultry hygiene is vertical and horizontal transmission of $S$. Enteritidis and $S$. Typhimurium without morbidity and mortality within the broiler production system [5], which impedes prompt action to prevent bacterial transmission to other healthy animals and food commodities.

In the context of extensive horizontal gene transfer events among diverse bacterial species, the emergence of NTS resistant to multiple antimicrobial agents in intensive animal farming poses a serious challenge to the treatment of severe bacterial infections. Antibiotics are used to treat clinical diseases but are routinely overused as low-cost substitutes for hygiene measures and as growth promoters in some countries [6]. Accumulating evidence linking antibiotics abuse in livestock with the emergence of bacterial resistance implicates a potential role of farm animals in the transmission of antibiotic resistance. Recent surveillance reports show that antibiotic-resistant NTS isolates are most frequently recovered from pigs and poultry, which rank first and second, respectively, in the global consumption of antibiotics per animal biomass [7, 8]. Furthermore, a high prevalence of resistance to ciprofloxacin and cephalosporins has been observed in Salmonella isolates from humans and poultry products in Korea and China, where these antibiotics are routinely used in largescale intensive husbandry systems [9-11].

In our previous study, we demonstrated that $S$. Typhimurium ST1120 isolated from swine feces contains multiple genes associated with virulence and antibiotic resistance in its genome and exhibited substantial invasion and intracellular survival abilities when added to host cells [12]. In order to improve our understanding of the prevalence of Salmonella in diverse livestock, S. Typhimurium ST1539 isolated from a duck slaughterhouse in Korea was subjected to whole genome sequencing, and its genetic loci associated with virulence and resistance were identified and compared with those of ST1120 in this study. S. Typhimurium ST1539, in agreement with the results of comparative genomic analysis, was more virulent than ST1120 in animal models but was more susceptible to antibiotics. Comparative genomics approaches to diverse Salmonella serotypes isolated from different livestock might provide insights into the mechanism of Salmonella adaptation in different host animals, as well as into the correlation between Salmonella serotypes and host preference.

\section{Materials and Methods}

\section{Bacterial Strains and Growth Conditions}

S. Typhimurium ST1539 was isolated from a duck slaughterhouse located in Yangpyeong, Gyeong-gi Province in Korea. S. Typhimurium ST1120 previously reported in our study was isolated from feces of pig [12]. All S. Typhimurium strains including LT2 [13], 14028s [14], and SL1344 [15] were grown in Luria Bertani (LB) broth (Difco, USA) or Mueller Hinton broth (Difco) at $37^{\circ} \mathrm{C}$ with shaking at $220 \mathrm{rpm}$.

\section{Genome Sequencing and Assembly}

S. Typhimurium ST1539 was cultivated in LB broth overnight. Bacterial cells were harvested by centrifugation at $16,000 \times g$ for $1 \mathrm{~min}$ and subjected to genomic DNA extraction using a GeneElute Bacterial Genomic DNA Kit (Sigma-Aldrich, USA). DNA concentration was estimated using a SimpliNano spectrophotometer (GE Healthcare, USA). A total of $5 \mu \mathrm{g}$ of isolated DNA was used for SMRT sequencing (Pacific Biosciences, USA), conducted at LabGenomics Inc. (Korea). A total of 1,644,445,258 bp after sequencing was subjected to de novo assembly using the hierarchical genome assembly process (HGAP, Version 2.3) workflow [16] with 214 folds of genome coverage. Finally, the circular forms of contigs were checked using MUMmer 3.5 [17] and self-similar ends were trimmed for manual genome closure.

\section{Genome Annotation and Analysis}

For gene annotation, acquired contigs were processed using Prokka version 1.12 [18], GeneMark [19], and NCBI BLASTP [20]. RNAmmer 1.2 [21] and tRNAscan-SE [22] were used for rRNA and tRNA gene predictions, respectively. Analyzed contigs were deposited in GenBank under accession numbers CP035301 (chromosome) and CP035302 (plasmid). The genome was mapped using DNAPlotter [23], including prophage regions identified using PHAge Search Tool (PHAST) [24]. A genome tree was created using JSpeciesWS based on average nucleotide identity (ANI) [25]. Salmonella in silico typing resource (SISTR) [26] was used to predict the serotype of ST1539. BL2SEQ [27] was used to perform comparative analysis and the result was visualized using Easyfig [28]. Antibiotic resistance-associated genes were predicted using the Comprehensive Antibiotic Resistance Database (CARD) [29]. Virulence-associated genes were analyzed using SPIFinder server ver. 1.0 [30] and the Virulence Factors Database (VFDB) [31].

\section{Pulsed-Field Gel Electrophoresis (PFGE) Analysis}

PFGE analysis was conducted according to the methods of Tenover et al. [32] and Wonderling et al. [33] with slight modifications. Salmonella cells embedded in agarose plugs were prepared as described in the previous study [34]. Salmonella colonies were collected from LB agar plates and suspended in TE suspension buffer (100 mM Tris and $100 \mathrm{mM}$ EDTA, pH 7.5). The turbidity of the bacterial cell suspension was adjusted to $20 \%$ transmittance using a colorimeter (bioMérieux, France). The cell 
suspension was mixed with proteinase $\mathrm{K}$ and $1.2 \%$ agarose (FMC Bioproducts, USA), and then dispensed into disposable plug molds (Bio-Rad, USA) containing ES buffer (0.5 M EDTA, pH 9.0; $1 \%$ sodium-lauroyl-sarcosine) and proteinase $\mathrm{K}$. The plugs were incubated in a water bath at $55^{\circ} \mathrm{C}$ for $1 \mathrm{~h}$ and then washed using sterile water and TE buffer (10 mM Tris and $1 \mathrm{mM}$ EDTA, pH 7.5) preheated to $50^{\circ} \mathrm{C}$. The washed plugs were cut into two $1-\mathrm{mm}-$ wide slices and then incubated with $\mathrm{XbaI}$ (Promega, USA) at $37^{\circ} \mathrm{C}$ for $3 \mathrm{~h}$. Digested DNAs in the plugs were separated using $1 \%$ agarose gel in the Contour Clamped Homogenous Field (CHEF) DR II electrophoresis system (Bio-Rad) at $14^{\circ} \mathrm{C}$. Salmonella serotype Braenderup H9812 (ATCC, USA) restricted with XbaI was used as the universal size standard. After electrophoresis, the gel stained using $50 \mathrm{mg} / \mathrm{ml}$ ethidium bromide was observed on a UV transilluminator. Macro-restriction patterns were analyzed using BioNumerics software (Applied-Maths, Sin-Martens-Latem, Belgium) based on Dice coefficients with a $1 \%$ band position tolerance.

\section{Antibiotic Susceptibility Test}

Disk diffusion assay was applied to test antibiotic susceptibility [35]. Briefly, Salmonella cells were cultured in Mueller Hinton broth at $37^{\circ} \mathrm{C}$ up to the McFarland turbidity standard of 0.5 . Sterile cotton swabs were used to spread bacterial cells evenly on Mueller Hinton agar plates. Disks containing antibiotics at indicated concentrations were placed onto the agar plates and incubated at $35^{\circ} \mathrm{C}$ for 16 to $18 \mathrm{~h}$. The diameter of the growth inhibition zone was measured and used to interpret resistance or susceptibility according to the CLSI standards [36].

\section{Lactate Dehydrogenase (LDH) Cytotoxicity Assay}

HeLa cells (ATCC) were seeded onto 96-well cell culture plates at a density of $1 \times 10^{4}$ cells per well and incubated in Dulbecco's modified Eagle's medium (DMEM; Gibco, USA) supplemented with $10 \%$ fetal bovine serum (FBS, Gibco) prior to bacterial infection. Salmonella cells cultivated in LB broth overnight were washed and resuspended in DMEM broth, and then added to HeLa cells at a multiplicity of infection (MOI) of 100. At $6 \mathrm{~h}$ postinfection, bacterial cytotoxicity was evaluated using CytoToxONE Homogeneous Membrane Integrity Assay (Promega) according to the manufacturer's instructions as described in the previous study [12]. LDH released from damaged HeLa cells converts lactate to pyruvate and produces $\mathrm{NADH}$, which in turn converts resazurin to the fluorescent compound resorufin. Fluorescence was measured using a fluorometer (BioTek Synergy HTX MultiMod Reader, USA). Intact HeLa cells not treated with Salmonella cells were used in parallel as a negative control.

\section{Invasion and Survival Assays}

Gentamycin protection assay was conducted to evaluate the ability of Salmonella to invade host cells and to survive inside host cells [12]. For the invasion assay, epithelial HeLa cells were seeded onto 24 -well cell culture plates at a density of $1 \times 10^{5}$ cells per well containing DMEM supplemented with $10 \%$ FBS prior to bacterial infection. Salmonella strains cultured in LB broth for $3 \mathrm{~h}$ were washed and resuspended in DMEM and added to HeLa cells at a MOI of 100 . At 30 min post-infection, the medium was replaced with fresh DMEM containing gentamicin at $100 \mu \mathrm{g} / \mathrm{ml}$ to inactivate extracellular bacteria, and infected cells were further incubated for $1.5 \mathrm{~h}$. HeLa cells were then lysed with $1 \%$ Triton X100 (Sigma-Aldrich), and the lysates were diluted and plated on LB agar plates in order to count the intracellular bacteria. For survival assay, macrophage-like RAW264.7 (ATCC) cells were seeded onto 24-well cell culture plates at a density of $2 \times 10^{5}$ cells per well, and Salmonella cells at stationary phase in LB broth were added to host cells at a MOI of 100 and incubated for $30 \mathrm{~min}$. After $1.5 \mathrm{~h}$ incubation with gentamicin $(100 \mu \mathrm{g} / \mathrm{ml})$ as described above, host cells were replenished with fresh DMEM containing gentamicin at $20 \mu \mathrm{g} / \mathrm{ml}$ for $9 \mathrm{~h}$. Intracellular Salmonella cells were counted in the same way as described in the invasion assay.

\section{Mouse Infection Experiment}

For mouse infection experiments, 7-week-old BALB/c female mice were used according to protocols approved by the Kangwon University Institute Animal Care and Use Committee (Permit number: KW-160201-1). A total of 24 mice were divided into 6 groups and infected intraperitoneally with $1 \times 10^{2}, 1 \times 10^{3}$, or $1 \times$ $10^{4}$ CFU of ST1539 or ST1120, respectively. Infected mice were monitored for two weeks and then euthanized according to the approved protocol.

\section{Statistical Analysis}

Every test was repeated at least three times using different bacterial colonies. Results were averaged and presented with their standard deviations. For statistics, Student's $t$-test was applied and $p$-values less than 0.05 were considered statistically significant.

\section{Results}

\section{Understanding General Genome Characteristics of $S$. Typhimurium ST1539}

Whole genome sequencing of S. Typhimurium ST1539 revealed two contigs constituting a circular chromosome of $4,905,039 \mathrm{bp}$ and a plasmid of 93,876 bp. The chromosome was predicted to contain a total of 4,327 ORFs including 4,219 coding sequences (CDSs), 22 rRNA genes, and 86 tRNA genes with a $52.15 \%$ GC content, whereas 96 ORFs estimated in the plasmid were all predicted as CDSs with a $53.11 \%$ GC content (Fig. 1). In view of genetic exchanges through bacteriophages, in silico PHAST analysis [24] of ST1539 genome sequences identified 6 prophages including PaV-LD, RE-2010, Gifsy-1, ST64B, Gifsy-2, and Tyrion (Fig. 1), three of which (PaV-LD, RE-2010, and Tyrion) are rarely detected in $S$. Typhimurium strains [37, 38]. In compliance with the presence of distinct prophages in the 
A

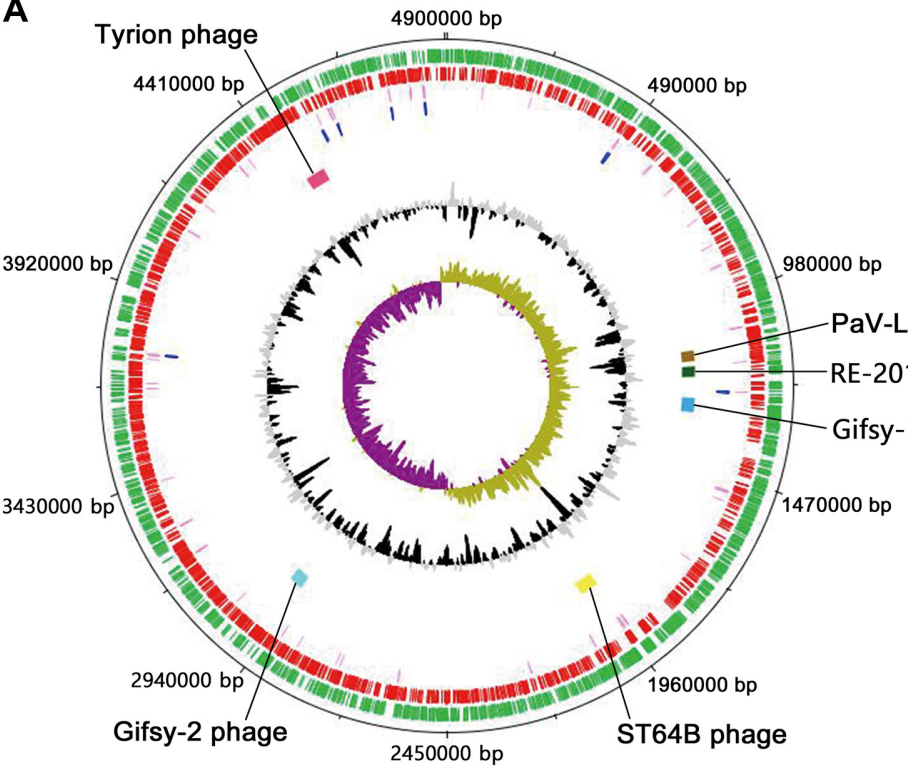

B

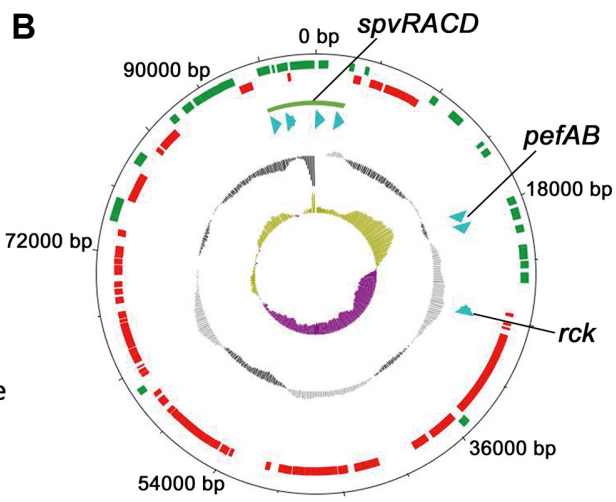

Fig. 1. Genome maps of $S$. Typhimurium ST1539.

The ST1539 genome that consists of the chromosome (A; 4,905,039 bp) and plasmid pST1539 (B; 93,876 bp) was mapped using DNAPlotter.

ST1539 genome, the phylogenetic tree analysis using ANI values proposed that ST1539 was not intimately associated with other $S$. Typhimurium strains, though it still belonged to a cluster of $S$. Typhimurium strains in comparison with other serotypes (Fig. 2). Serotyping of ST1539 determined it to be Typhimurium by a serological test based on Kauffmann-White scheme [39] and via in silico prediction using SISTR [26]. ST1539 also showed a distinct XbaI-PFGE pattern from other $S$. Typhimurium strains such as LT2 and 14028s in PFGE analysis (Fig. S1). ST1539 was fused with other $S$. Typhimurium strains at a higher distance of $79.4 \%$ in a dendrogram calculated with Dice coefficients, while ST1120 had a 95.7\% sequence similarity with LT2. ST1120 reported in our previous study (GenBank Acc. No.

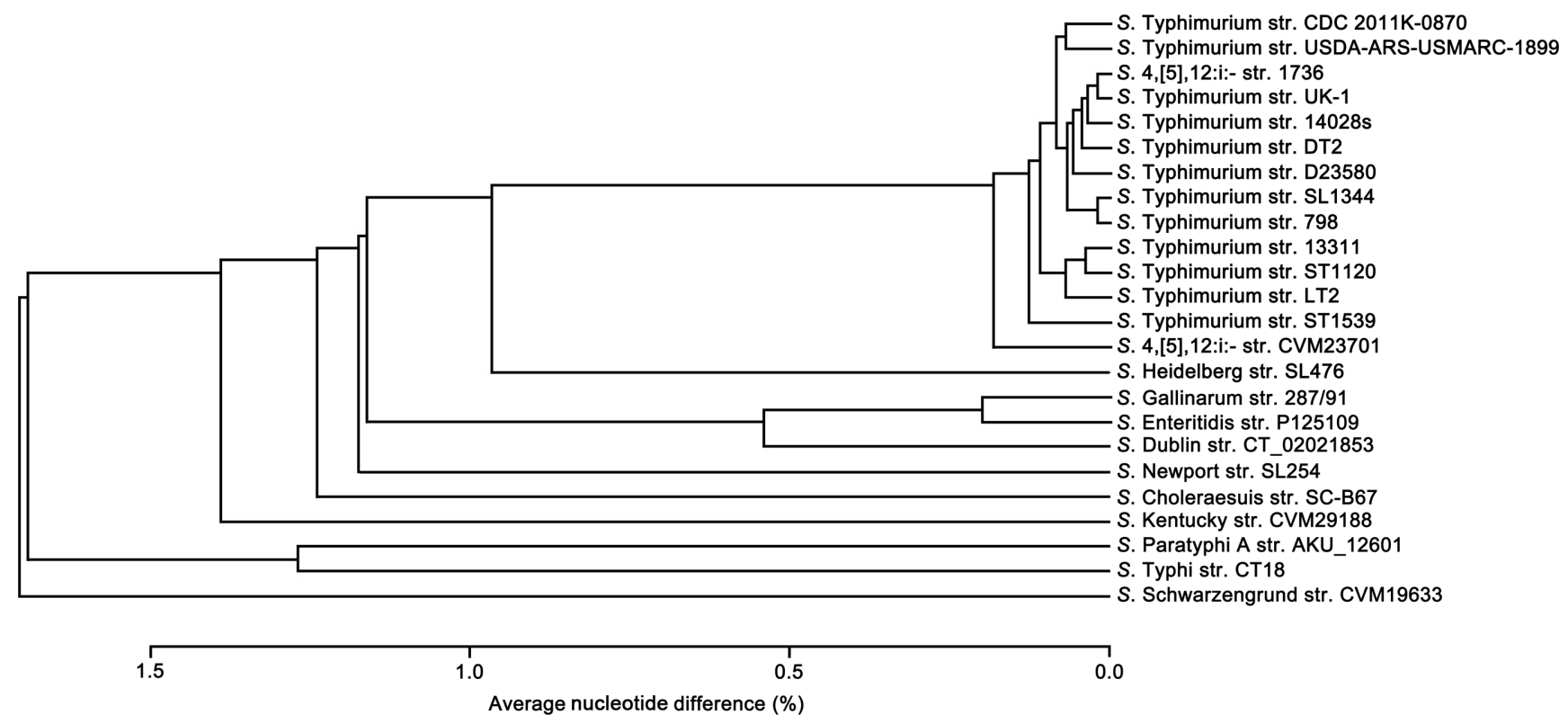

Fig. 2. Phylogenetic tree analysis of $S$. Typhimurium ST1539.

The genome sequence of ST1539 was compared to those of other Salmonella serotypes by ANI using JSpeciesWS. 
CP021909.1 and CP021910.1; [12]) is a S. Typhimurium strain isolated from swine feces in Korea. In an effort to understand the strategies of $S$. Typhimurium in adapting to diverse livestock, the strains ST1539 and ST1120 with a genetic divergence in the serogroup Typhimurium were further investigated in terms of their genetic determinants associated with virulence and antibiotic resistance.

\section{Evaluation of Antibiotic Resistance of S. Typhimurium ST1539}

In our previous study, we showed that ST1120 was more resistant to several antibiotics such as streptomycin, chloramphenicol, and ampicillin than other $S$. Typhimurium strains LT2, 14028s, and SL1344, and we observed a large number of genes in its genome that were presumably responsible for the resistance properties [12]. Considering that piggery and poultry farms are the top 2 in antibiotic use in animal farming, ST1539 was likely to develop tolerance against antibiotics extensively used in poultry farming. However, the in silico comparative analysis with ST1120 using CARD predicted that ST1539 lacked multiple genes encoding components of efflux pumps, which play an important role in conferring resistance by actively excreting the harmful antibiotic drugs from the bacteria (Table 1). When the two isolates were treated with 10 different antibiotics, ST1539 exhibited susceptibility to all the tested antibiotics according to the CLSI standards and was more susceptible than ST1120 specifically to gentamicin, kanamycin, and neomycin (Fig. 3 and Table S1). ST1120 isolated from a pig farm was somewhat resistant to six

Table 1. CDCs predicted to be associated with antimicrobial resistance.

\begin{tabular}{ll}
\hline \multicolumn{1}{c}{ ARO $^{\text {a }}$ category } & \multicolumn{1}{c}{ ST1539 Locus_tag } \\
\hline Efflux pump complex or & ST1539_0378, ST1539_1019, ST1539_1021, ST1539_1807, \\
subunit conferring antibiotic & ST1539_1918, ST1539_2111, ST1539_2877, ST1539_2953, \\
resistance & ST1539_3114, ST1539_3335, ST1539_3336, ST1539_3456, \\
& ST1539_3458, ST1539_3459, ST1539_3460, ST1539_4395
\end{tabular}

ST1120 Locus_tag
ST1120_01136, ST1120_01137, ST1120_02874,
ST1120_02875, ST1120_00207, ST1120_02873,
ST1120_02870, ST1120_01647, ST1120_00208,
ST1120_01138, ST1120_02872, ST1120_04131,
ST1120_04132, ST1120_01684, ST1120_01685,
ST1120_03550, ST1120_03551, ST1120_03958,
ST1120_00647, ST1120_02167, ST1120_04203,
ST1120_01279, ST1120_01724, ST1120_04342,
ST1120_00707, ST1120_01277, ST1120_02263,
ST1120_01899, ST1120_03223, ST1120_01140,
ST1120_03925, ST1120_02871, ST1120_01810,
ST1120_01911, ST1120_02695, ST1120_03549,
ST1120_00711, ST1120_00978, ST1120_01366,
ST1120_02503, ST1120_00010, ST1120_02757,
ST1120_02428, ST1120_03007, ST1120_04535,
ST1120_01260, ST1120_01261
ST1120_02466, ST1120_03558
ST1120_01481, ST1120_02368
ST1120_02584, ST1120_02215
N/D
N/D
ST1120_01961
N/D
ST1120_00256
ST1120_00771
N/D
ST1120_03945
ST1120_02823, ST1120_03041, ST1120_03042,
ST1120_00428
ST1120_00839

\begin{tabular}{lll} 
Aminocoumarin resistance & N/D & ST1120_02466, ST1120_03558 \\
Aminoglycoside resistance & ST1539_2243 & ST1120_01481, ST1120_02368 \\
Beta-lactam resistance & N/D & ST1120_02584, ST1120_02215 \\
Cephamycin resistance & ST1539_3699 & N/D \\
Elfamycin resistance & ST1539_0399, ST1539_4317 & N/D \\
Fluoroquinolone resistance & ST1539_2345, ST1539_2346, ST1539_4143, ST1539_41444 & ST1120_01961 \\
Fosfomycin resistance & ST1539_1539 & N/D \\
Isoniazid resistance & N/D & ST1120_00256 \\
Mupirocin resistance & N/D & ST1120_00771 \\
Nitrofuran resistance & ST1539_2946 & N/D \\
Peptide antibiotic resistance & N/D & ST1120_03945 \\
Polymyxin resistance & N/D & ST1120_02823, ST1120_03041, ST1120_03042, \\
Sulfonamide resistance & N/D & ST1120_00428 \\
\hline
\end{tabular}

${ }^{a} A R O$ (antibiotic resistance ontology) analyzed by Resistance Gene Identifier (RGI) according to CARD (comprehensive antibiotic resistance database). N/D, Not detected. 


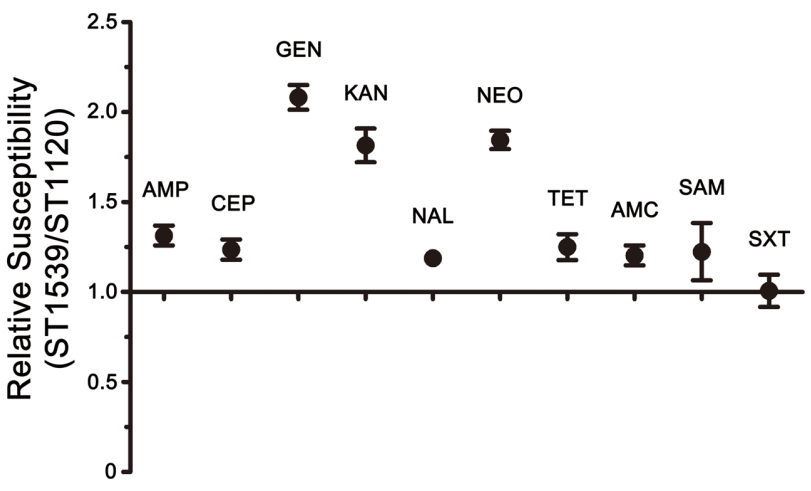

Fig. 3. Comparison of antibiotic resistance profiles between ST1539 and ST1120.

Antibiotic susceptibility test of ST1539 and ST1120 was conducted on Mueller Hinton agar plates using antibiotic disks. Diameters of growth inhibition zones were measured and used to calculate relative susceptibility between ST1539 and ST1120. The ratios from three independent assays were averaged and plotted. The antibiotics tested were AMP, ampicillin; CEP, cephalothin; GEN, gentamicin; KAN, kanamycin; NAL, naladixic acid; NEO, neomycin; TET, tetracycline; AMC, amoxicillin/clavulanic acid; SAM, ampicillin/sulbactam; SXT, sulfamethoxazole/trimethoprim. The concentration of each antibiotic was determined according to the CLSI standards for testing against Enterobacteriaceae family.

antibiotics and especially exhibited higher resistance to a class of aminoglycosides including gentamicin, kanamycin, and neomycin, which reflects the overuse of aminoglycoside antibiotics in pig farms ever since the mid-1980s in Korea $[40,41]$. In the case of $\beta$-lactam antibiotics, ST1120 showed an intermediate level of resistance against ampicillin, but both isolates ST1120 and ST1539 were determined to be susceptible to the combinations (amoxicillin/clavulanic acid and ampicillin/sulbactam) supplemented with $\beta$ lactamase inhibitors. Taken together, the pig farm isolate ST1120 was likely to develop resistance against $\beta$-lactams and aminoglycosides due to frequent exposure to these antibiotics. According to a recent surveillance on antibiotic resistance in poultry commodities in Korea, Salmonella isolates from duck meat showed much lower resistance to a variety of antibiotics, including ampicillin, tetracycline, and nalidixic acid, than those from chicken meat [42], which can be attributed to varying antibiotic usage across poultry species. A statistical analysis using a large number of Salmonella isolates would define the correlation between antibiotic consumption levels and antibiotic resistance prevalence in livestock.

\section{Exploring Virulence Determinants in $S$. Typhimurium ST1539 Genome}

The potential virulence of ST1539 was assessed by searching for virulence-associated determinants in the genome (Table 2). Salmonella pathogenicity islands (SPIs) are the best known genetic loci critical for Salmonella virulence. Virulence effectors encoded by SPIs are translocated to the cytosol of host cells through bacterial type III secretion systems (T3SSs) and manipulate host cellular functions for bacterial invasion and proliferation inside hosts [43, 44]. Eight clusters of SPI-1, SPI-2, SPI-3, SPI-4, SPI-5, SPI-12, SPI-13, and SPI-14 were all found in both ST1539 and ST1120 (Table 2).

Bacteriophage-mediated horizontal gene transfer diversifies

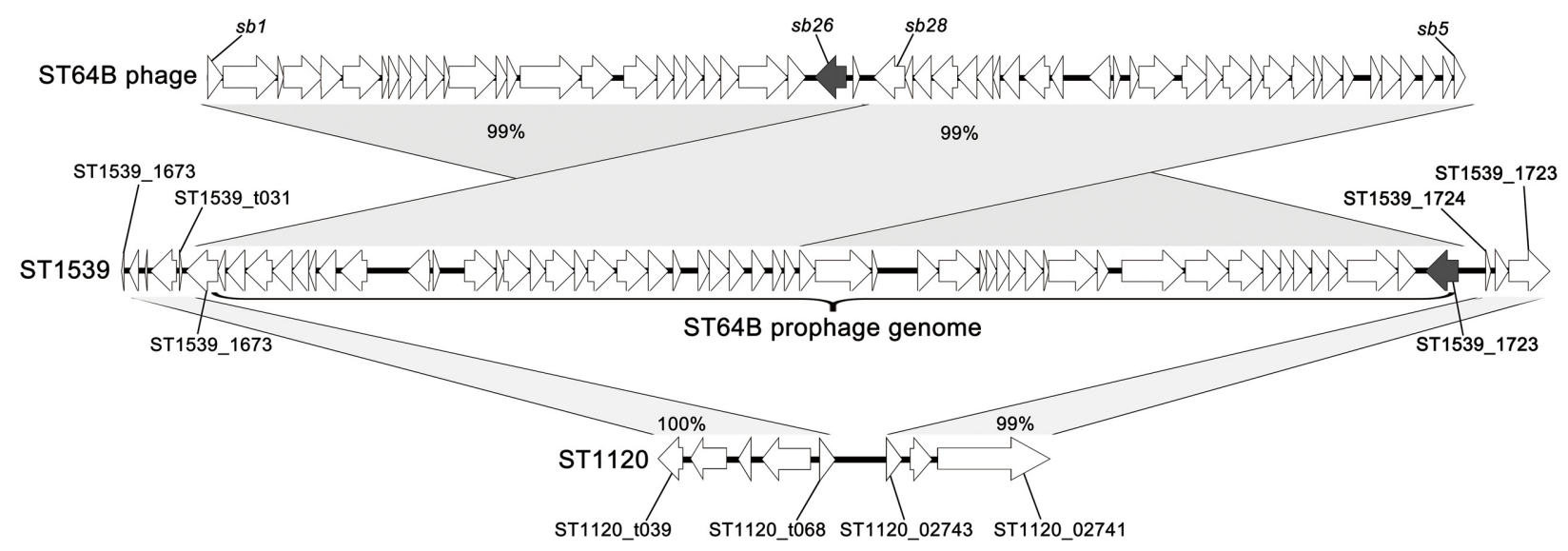

Fig. 4. Sequence alignment of ST64B phage with ST539 and ST1120.

The ST64B prophage region of S. Typhimurium ST1539 (middle) was compared with those of ST64B phage (GenBank Acc. No. AY055382; top) and S. Typhimurium ST1120 (bottom) using Easyfig. Gene sb26 and its homolog ST1539_1723 are indicated with grey arrows within the phage ST64B (top) and prophage ST64B (middle), respectively. 
bacterial genomic repertoires and enables them to adapt to environmental changes efficiently during host infections by integrating virulence-associated genes into the genome [45, 46]. In comparison with other $S$. Typhimurium strains such as LT2 and ST1120, ST1539 possesses the prophage ST64B, which was first identified in S. Typhimurium DT 64 (Fig. 4) [47]. ST64B prophage covers a 40,149-bp region containing 51 CDSs from ST1539_1673 to ST1539_1723, and its role

Table 2. Virulence factors in silico predicted in ST1539 and ST1120.

\begin{tabular}{|c|c|c|c|}
\hline Virulence factor & ST1539 Locus tag (gene) & ST1120 Locus tag (gene) & Function \\
\hline${ }^{a,+}$ SPI-1 & ST1539_0869 (invH) - ST1539_0901 (avrA) & ST1120_03592 (avrA) - ST1120_03632 (invH) & Salmonella pathogenicity island 1 \\
\hline${ }^{\mathrm{a},+} \mathrm{SPI}-2$ & ST1539_2241 (ssaU) - ST1539_2271 (ssrB) & ST1120_02123 (ssrB) - ST1120_02166 (ssaU) & Salmonella pathogenicity island 2 \\
\hline${ }^{a,+}$ SPI-3 & ST1539_0074 (mgtC) - ST1539_0085 & ST1120_04484 - ST1120_04497 (mgtC) & Salmonella pathogenicity island 3 \\
\hline${ }^{a,+}$ SPI-4 & ST1539_3811 (siiF) - ST1539_3815 (siiA) & ST1120_00391 (siiA) - ST1120_00398 (siiF) & Salmonella pathogenicity island 4 \\
\hline${ }^{a,+}$ SPI-5 & ST1539_2542(copR) - ST1539_2549 (pipA) & ST1120_01833 (pipA) - ST1120_01841 (copR) & Salmonella pathogenicity island 5 \\
\hline${ }^{\mathrm{a}, \mathrm{t}} \mathrm{SPI}-13$ & ST1539_0658 (exuT) - ST1539_0674 & ST1120_03852 - ST1120_03871 (exuT) & Salmonella pathogenicity island 13 \\
\hline${ }^{\mathrm{a}, \mathrm{t}} \mathrm{SPI}-14$ & ST1539_2724 - ST1539_2731 & ST1120_01634 - ST1120_01640 & Salmonella pathogenicity island 14 \\
\hline${ }^{\mathrm{a},{ }^{\dagger} \mathrm{C}} 63 \mathrm{PI}$ & ST1539_1265 - ST1539_1271 & ST1120_03251 - ST1120_03258 & Centisome 63 pathogenicity island \\
\hline${ }^{\text {at }}$ effectors & $\begin{array}{l}\text { ST1539_0828 (sopD), } \\
\text { ST1539_1018 (pipB2), } \\
\text { ST1539_1202 (sseB), } \\
\text { ST1539_1204 (gogB), } \\
\text { ST1539_1476 (sseL), } \\
\text { ST1539_1520 (sspH1), } \\
\text { ST1539_1618 (sseK2), } \\
\text { ST1539_1685 (sopA), } \\
\text { ST1539_1723 (sseK3), } \\
\text { ST1539_2064 (steC), } \\
\text { ST1539_2090 (steA), } \\
\text { ST1539_2135 (sseJ), } \\
\text { ST1539_2164 (sifB), } \\
\text { ST1539_2306 (steB), } \\
\text { ST1539_2532 (sifA), } \\
\text { ST1539_2660 (sopD2), } \\
\text { ST1539_2903 (slrP), } \\
\text { ST1539_2903 (slrP), } \\
\text { ST1539_4130 (sseK1) }\end{array}$ & $\begin{array}{l}\text { ST1120_03677 (sopD), } \\
\text { ST1120_03515 (pipB2), } \\
\text { ST1120_03273(sseb), } \\
\text { ST1120_03322 (gogB), } \\
\text { ST1120_03030 (sseL), } \\
\text { ST1120_02984 (sspH1), } \\
\text { ST1120_02880 (sseK2), } \\
\text { ST1120_02809 (sopA), } \\
\text { N/D, } \\
\text { ST1120_02449 (steC), } \\
\text { ST1120_02332 (steA), } \\
\text { N/D, } \\
\text { ST1120_02351 (sifB), } \\
\text { ST1120_02378 (steB), } \\
\text { ST1120_01970 (sifA), } \\
\text { ST1120_01712 (sopD2), } \\
\text { ST1120_01575 (slrP), } \\
\text { ST1120_01575 (slrP), } \\
\text { ST1120_00294 (sseK1) }\end{array}$ & T3SS effectors \\
\hline${ }^{\mathrm{b}, \mathrm{t}}$ lpf operon & ST1539_0209 (lpfA) - ST1539_0214 (lpfE) & ST1120_04370 (lpfE) - ST1120_04374 (lpfA) & Long polar fimbriae \\
\hline${ }^{b, t} \mathrm{csg}$ operon & ST1539_2605 (csgC) - ST1539_2611 (csgG) & ST1120_01884 (csgG) - ST1120_01890 (csgC) & Curli fimbriae \\
\hline${ }^{\mathrm{b}, \mathrm{t}}$ fim operon & ST1539_3130 (fimF) - ST1539_3134 (fimI) & ST1120_01329 (fimI) - ST1120_01333 (fimF) & Type 1 fimbriae \\
\hline${ }^{\mathrm{b}, t} b c f$ operon & ST1539_3641 (bcfG) - ST1539_3647 (bcfA) & $\mathrm{N} / \mathrm{D}$ & Fimbriae \\
\hline${ }^{\mathrm{b},{ }^{+}}$mig-14 & ST1539_1017 & ST1120_03517 & $\begin{array}{l}\text { Antimicrobial peptide resistance } \\
\text { protein }\end{array}$ \\
\hline${ }^{\mathrm{b}, \dagger}$ ompA & ST1539_2677 & ST1120_01817 & Outer membrane protein $\mathrm{A}$ \\
\hline${ }^{\mathrm{a}, \mathrm{f}}$ spv operon & $\begin{array}{l}\text { ST1539_p001 (spvC), ST1539_p002 (spvD), } \\
\text { ST1539_p091 (spvR), ST1539_p093 (spvA) }\end{array}$ & $\mathrm{N} / \mathrm{D}$ & $\begin{array}{l}\text { Plasmid encoded virulence } \\
\text { proteins }\end{array}$ \\
\hline${ }^{\mathrm{a}, \pm}$ pef operon & ST1539_p017(pefA), ST1539_p018(pefB) & $\mathrm{N} / \mathrm{D}$ & Plasmid encoded fimbriae \\
\hline${ }^{\mathrm{a}, \mathrm{t}} r c k$ & ST1539_p025 & $\mathrm{N} / \mathrm{D}$ & Plasmid encoded invasin \\
\hline
\end{tabular}

${ }^{a}$ Identified by SPIFinder ver.1.0.

${ }^{b}$ Identified by Virulence Factors DataBase.

${ }^{\dagger}$ Chromosome of ST1539, ST1120.

${ }^{\ddagger}$ Plasmid of ST1539.

N/D, Not detected. 
associated with $S$. Typhimurium fitness has been demonstrated in $S$. Typhimurium isolates from human blood samples [48]. The prophage region was strongly conserved in $S$. Typhimurium strains isolated from blood samples and its presence favored bacterial growth in human blood and plasma. SseK3 encoded by sb26 (homologous to ST1539_1723) within the phage ST64B is a member of SseK/NleB effector proteins. Its expression is co-regulated with other SPI-2 genes by SsrB, a primary transcriptional regulator of SPI-2, and SseK3 is translocated into the host cytosol by SPI-2 T3SS [49].

Aside from the ST64B prophage, ST1539 encompasses multiple virulence-relevant determinants encoding SseJ effector, SpvC/SpvD effectors, fimbriae ( $b c f$ operon and pef $A B$ ), and Rck invasin (Table 2). Some of these genes including $s p v C D$, pef $A B$, and $r c k$ are uniquely located in the plasmid of ST1539 (Fig. 1 and Table 2).

\section{Assessment of Virulence of S. Typhimurium ST1539 In Vitro and In Vivo}

Comparative genomic analysis between ST1539 and ST1120 predicted more virulence-associated genetic features in ST1539 (Table 2). We previously observed that ST1120 was competent to invade into host epithelial cells and to replicate inside macrophages when compared with other virulent $S$. Typhimurium strains such as 14028 s and SL1344 [12]. The virulence of ST1539 was compared with that of ST1120 to verify the in silico prediction. When epithelial HeLa cells were treated with $S$. Typhimurium strains (LT2, 14028s, SL1344, ST1120, and ST1539), ST1539 had the highest cytotoxic activity to the host cells (Fig. 5A), whereas all strains except LT2 showed comparable invasion ability
A

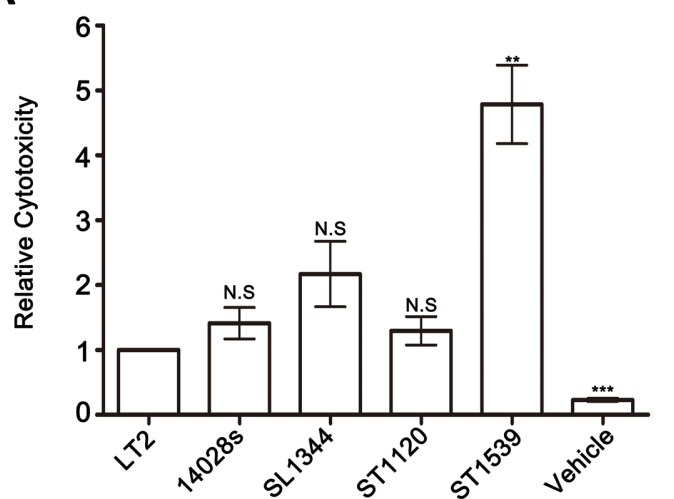

B

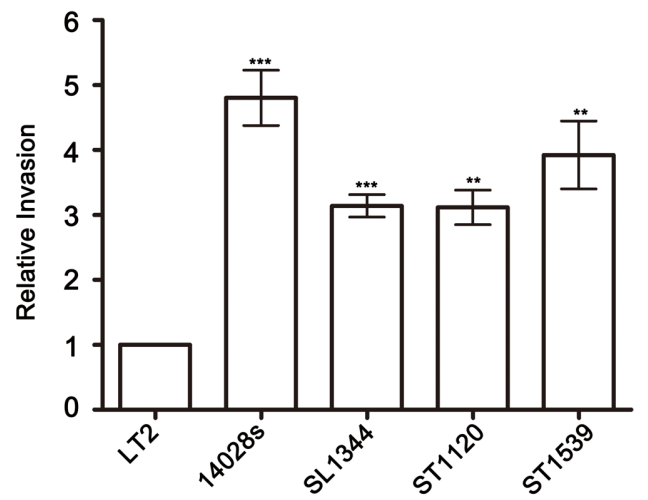

C

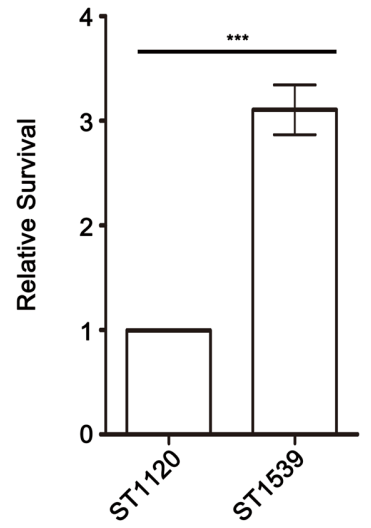

D

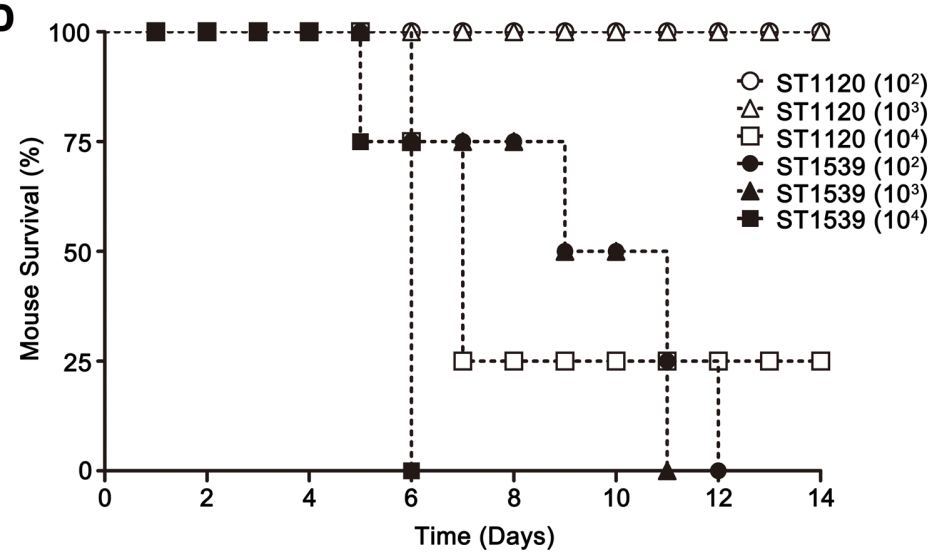

Fig. 5. Virulence comparison in vitro and in vivo between ST1539 and ST1120.

Cytotoxicity (A) and invasion ability (B) of ST1539 were compared with those of other S. Typhimurium strains (LT2, 14028s, SL1344, and ST1120) using epithelial HeLa cells. The levels of cytotoxicity and invasion ability of each strain were compared with those of LT2 and the ratios were plotted. Differences with $p$-values less than $0.05\left(^{* *}\right)$ or $\left.0.01{ }^{* * *}\right)$ in comparison with LT2 were denoted with asterisks. N.S. indicates no significance in comparison with the control. Survival ability between ST1539 and ST1120 was compared using macrophage-like RAW264.7 cells (C). Asterisks indicate a difference of $p$-value less than 0.01 between the two strains. For animal tests (D), BALB/c mice were intraperitoneally infected with ST1539 and ST1120 at different doses $\left(10^{2}, 10^{3}\right.$, and $10^{4} \mathrm{CFU} /$ mouse) and their survival rates were plotted for 2 weeks. 
(Fig. 5B). The attenuated virulence of LT2 has been previously reported in vitro and in vivo elsewhere [50, 51]. In the survival assay using RAW264.7 macrophage-like cells, ST1539 replicated faster than ST1120 inside host cells, showing 1.5-fold higher cell numbers at $9 \mathrm{~h}$ post-infection (Fig. 5C), which might be attributable to multiple virulence determinants present in ST1539. The ability to persist and replicate inside macrophages is crucial in developing systemic infections in host animals [52]. We further compared the ability to conquer host animals between ST1539 and ST1120 (Fig. 5D). Mice intraperitoneally infected with ST1120 at $10^{3} \mathrm{CFU} /$ mouse survived during 14 days of observation but ST1539 killed all mice even at a lower dose of $10^{2} \mathrm{CFU} /$ mouse, indicating its hypervirulence in vivo. These results are consistent with the in silico analysis results demonstrating multiple virulence attributes in the ST1539 genome.

In summary, we aimed to characterize the properties of ST1539 in the context of antimicrobial resistance and virulence based on the genome sequences. Its resistance and virulence were compared with those of ST1120, which was also isolated from livestock in Korea. Data about the genome properties of Salmonella isolates would provide insights into the evolutionary adaptation process of Salmonella during environmental changes in the livestock industry, as well as help find a way to manage Salmonella infections transmitted via contaminated foods of animal origin.

\section{Acknowledgments}

This research was supported by a grant of the National Research Foundation of Korea (NRF), funded by the Ministry of Science and ICT (grant number: NRF-2017R1A2B4003834) and a grant supported by the Ministry of Food and Drug Safety (14162MFDS972), Republic of Korea.

\section{Conflict of Interest}

The authors have no financial conflicts of interest to declare.

\section{References}

1. Chen H-M, Wang Y, Su L-H, Chiu C-H. 2013. Nontyphoid Salmonella infection: microbiology, clinical features, and antimicrobial therapy. Pediatr. Neonatol. 54: 147-152.

2. Crump JA, Sjölund-Karlsson M, Gordon MA, Parry CM. 2015. Epidemiology, clinical presentation, laboratory diagnosis, antimicrobial resistance, and antimicrobial management of invasive Salmonella infections. Clin. Microbiol. Rev. 28: 901937.

3. Jones TF, Ingram LA, Cieslak PR, Vugia DJ, Tobin-D'Angelo M, Hurd S, et al. 2008. Salmonellosis outcomes differ substantially by serotype. J. Infect. Dis. 198: 109-114.

4. Jackson BR, Griffin PM, Cole D, Walsh KA, Chai SJ. 2013. Outbreak-associated Salmonella enterica serotypes and food commodities, United States, 1998-2008. Emerg. Infect. Dis. 19: 1239-1244.

5. Barrow P, Huggins M, Lovell M, Simpson J. 1987. Observations on the pathogenesis of experimental Salmonella typhimurium infection in chickens. Res.Vet. Sci. 42: 194-199.

6. McEwen SA, Fedorka-Cray PJ. 2002. Antimicrobial use and resistance in animals. Clin. Infect. Dis. 34: S93-S106.

7. Van Boeckel TP, Brower C, Gilbert M, Grenfell BT, Levin SA, Robinson TP, et al. 2015. Global trends in antimicrobial use in food animals. Proc. Natl. Acad. Sci. USA 112: 5649-5654.

8. Hopkins S, Muller-Pebody B. 2015. UK one health report: joint report on human and animal antibiotic use, sales and resistance, 2013.

9. Yoon R-H, Cha S-Y, Wei B, Roh J-H, Seo H-S, Oh J-Y, et al. 2014. Prevalence of Salmonella isolates and antimicrobial resistance in poultry meat from South Korea. J. Food Prt. 77: 1579-1582.

10. Yang B, Cui Y, Shi C, Wang J, Xia X, Xi M, et al. 2014. Counts, serotypes, and antimicrobial resistance of Salmonella isolates on retail raw poultry in the People's Republic of China. J. Food Prot. 77: 894-902.

11. Antunes P, Mourão J, Campos J, Peixe L. 2016. Salmonellosis: the role of poultry meat. Clin. Microbiol. Infect. 22: 110-121.

12. Kim S, Kim E, Park S, Hahn TW, Yoon H. 2017. Genomic approaches for understanding the characteristics of Salmonella enterica subsp. enterica Serovar Typhimurium ST1120, Isolated from Swine Feces in Korea. J. Microbiol. Biotechnol. 27: 19831993.

13. McClelland M, Sanderson KE, Spieth J, Clifton SW, Latreille P, Courtney L, et al. 2001. Complete genome sequence of Salmonella enterica serovar Typhimurium LT2. Nature 413: 852.

14. Jarvik T, Smillie C, Groisman EA, Ochman H. 2010. Shortterm signatures of evolutionary change in the Salmonella enterica serovar Typhimurium 14028 genome. J. Bacteriol. 192: $560-567$.

15. Kröger C, Dillon SC, Cameron AD, Papenfort K, Sivasankaran SK, Hokamp K, et al. 2012. The transcriptional landscape and small RNAs of Salmonella enterica serovar Typhimurium. Proc. Natl. Acad. Sci. USA 109: E1277-E1286.

16. Chin C-S, Alexander DH, Marks P, Klammer AA, Drake J, Heiner C, et al. 2013. Nonhybrid, finished microbial genome assemblies from long-read SMRT sequencing data. Nat. Methods 10: 563-569. 
17. Kurtz S, Phillippy A, Delcher AL, Smoot M, Shumway M, Antonescu C, Salzberg SL. 2004. Versatile and open software for comparing large genomes. Genome Biol. 5: R12.

18. Seemann T. 2014. Prokka: rapid prokaryotic genome annotation. Bioinformatics 30: 2068-2069.

19. Lukashin AV, Borodovsky M. 1998. GeneMark. hmm: new solutions for gene finding. Nucleic Acids Res. 26: 1107-1115.

20. Altschul SF, Gish W, Miller W, Myers EW, Lipman DJ. 1990. Basic local alignment search tool. J. Mol. Biol. 215: 403-410.

21. Lagesen K, Hallin P, Rodland EA, Staerfeldt HH, Rognes T, Ussery DW. 2007. RNAmmer: consistent and rapid annotation of ribosomal RNA genes. Nucleic Acids Res. 35: 3100-3108.

22. Lowe TM, Eddy SR. 1997. tRNAscan-SE: a program for improved detection of transfer RNA genes in genomic sequence. Nucleic Acids Res. 25: 955-964.

23. Carver T, Thomson N, Bleasby A, Berriman M, Parkhill J. 2008. DNAPlotter: circular and linear interactive genome visualization. Bioinformatics 25: 119-120.

24. Zhou Y, Liang Y, Lynch KH, Dennis JJ, Wishart DS. 2011. PHAST: a fast phage search tool. Nucleic Acids Res. 39: W347-352.

25. Richter M, Rosselló-Móra R, Oliver Glöckner F, Peplies J. 2015. JSpeciesWS: a web server for prokaryotic species circumscription based on pairwise genome comparison. Bioinformatics 32: 929-931.

26. Yoshida CE, Kruczkiewicz P, Laing CR, Lingohr EJ, Gannon VP, Nash JH, et al 2016. The Salmonella in silico typing resource (SISTR): an open web-accessible tool for rapidly typing and subtyping draft Salmonella genome assemblies. PLoS One 11: e0147101.

27. Tatusova TA, Madden TL. 1999. BLAST 2 Sequences, a new tool for comparing protein and nucleotide sequences. FEMS Microbiol. Lett. 174: 247-250.

28. Sullivan MJ, Petty NK, Beatson SA. 2011. Easyfig: a genome comparison visualizer. Bioinformatics 27: 1009-1010.

29. Jia B, Raphenya AR, Alcock B, Waglechner N, Guo P, Tsang KK, et al. 2016. CARD 2017: expansion and model-centric curation of the comprehensive antibiotic resistance database. Nucleic Acids Res. 45: D566-D573.

30. Kozyreva VK, Crandall J, Sabol A, Poe A, Zhang P, Concepción-Acevedo J, et al. 2016. Laboratory investigation of Salmonella enterica serovar Poona outbreak in California: comparison of pulsed-field gel electrophoresis (PFGE) and whole genome sequencing (WGS) results. PLoS Curr. 8.

31. Chen L, Yang J, Yu J, Yao Z, Sun L, Shen Y, Jin Q. 2005. VFDB: a reference database for bacterial virulence factors. Nucleic Acids Res. 33: D325-D328.

32. Tenover FC, Arbeit RD, Goering RV, Mickelsen PA, Murray BE, Persing DH, Swaminathan B. 1995. Interpreting chromosomal DNA restriction patterns produced by pulsedfield gel electrophoresis: criteria for bacterial strain typing. J. Clin. Microbiol. 33: 2233-2239.
33. Wonderling L, Pearce R, Wallace FM, Call JE, Feder I, Tamplin M, et al. 2003. Use of pulsed-field gel electrophoresis to characterize the heterogeneity and clonality of Salmonella isolates obtained from the carcasses and feces of swine at slaughter. Appl. Environ. Microbiol. 69: 4177-4182.

34. Seo Y-S, Lee S-H, Shin E-K, Kim S-J, Jung R, Hahn T-W. 2006. Pulsed-field gel electrophoresis genotyping of Salmonella gallinarum and comparison with random amplified polymorphic DNA. Vet. Microbiol. 115: 349-357.

35. Hudzicki J. 2009. Kirby-Bauer disk diffusion susceptibility test protocol.

36. Reller LB, Weinstein M, Jorgensen JH, Ferraro MJ. 2009. Antimicrobial susceptibility testing: a review of general principles and contemporary practices. Clin. Infect. Dis. 49: 1749-1755.

37. Mottawea W, Duceppe M-O, Dupras AA, Usongo V, Jeukens J, Freschi L, et al. 2018. Salmonella enterica prophage sequence profiles reflect genome diversity and can be used for high discrimination subtyping. Front. Microbiol. 9:836.

38. Oladeinde A, Cook K, Orlek A, Zock G, Herrington K, Cox N, et al. 2018. Hotspot mutations and ColE1 plasmids contribute to the fitness of Salmonella Heidelberg in poultry litter. PLoS One 13: $\mathrm{e} 0202286$.

39. Popoff MY, Bockemühl J, Gheesling LL. 2004. Supplement 2002 (no. 46) to the Kauffmann-White scheme. Res. Microbiol. 155: $568-570$.

40. LIm S-K, Nam H-M, Lee H-S, Kim A-R, Jang G-C, Jung S-C, Kim T-S. 2013. Prevalence and characterization of apramycinresistant Salmonella enterica serotype Typhimurium isolated from healthy and diseased pigs in Korea during 1998 through 2009. J. Food Prot. 76: 1443-1446.

41. Cho S-H, Lim Y-S, Kang Y-H. 2012. Comparison of antimicrobial resistance in Escherichia coli strains isolated from healthy poultry and swine farm workers using antibiotics in Korea. Osong Public Health Res. Perspect. 3: 151-155.

42. APQA. 2017. Antimicrobial consumption in livestock and Monitoring of antimicrobial resistance in animals and carcasses, 2017. pp75-76. Animal and Plant Quarantine Agency, Gimcheon-si, Gyeongsangbuk-do.

43. Hensel M, Shea JE, Waterman SR, Mundy R, Nikolaus T, Banks G, et al. 1998. Genes encoding putative effector proteins of the type III secretion system of Salmonella pathogenicity island 2 are required for bacterial virulence and proliferation in macrophages. Mol. Microbiol. 30: 163-174.

44. Lostroh CP, Lee CA. 2001. The Salmonella pathogenicity island-1 type III secretion system. Microbes Infect. 3: 12811291.

45. Casjens S. 2003. Prophages and bacterial genomics: what have we learned so far? Mol. Microbiol. 49: 277-300.

46. Ochman H, Lawrence JG, Groisman EA. 2000. Lateral gene transfer and the nature of bacterial innovation. Nature 405: 299-304. 
47. Mmolawa PT, Willmore R, Thomas CJ, Heuzenroeder MW. 2002. Temperate phages in Salmonella enterica serovar Typhimurium: implications for epidemiology. Int. J. Med. Microbiol. 291: 633-644.

48. Herrero-Fresno A, Leekitcharoenphon P, Hendriksen RS, Olsen JE, Aarestrup FM. 2014. Analysis of the contribution of bacteriophage ST64B to in vitro virulence traits of Salmonella enterica serovar Typhimurium. J. Med. Microbiol. 63: 331-342.

49. Brown NF, Coombes BK, Bishop JL, Wickham ME, Lowden MJ, Gal-Mor O, et al. 2011. Salmonella phage ST64B encodes a member of the SseK/NleB effector family. PLoS One 6: e17824.

50. Swords WE, Cannon BM, Benjamin W. 1997. Avirulence of LT2 strains of Salmonella typhimurium results from a defective rpoS gene. Infect. Immun. 65: 2451-2453.

51. Wilmes-Riesenberg MR, Foster JW, Curtiss R. 1997. An altered rpoS allele contributes to the avirulence of Salmonella typhimurium LT2. Infect. Immun. 65: 203-210.

52. Chappell L, Kaiser P, Barrow P, Jones MA, Johnston C, Wigley P. 2009. The immunobiology of avian systemic salmonellosis. Vet. Immun. Immunopathol. 128: 53-59. 